\title{
Secure Data Transmission Based on Adaptive Chattering-Free Sliding Mode Synchronization of Unified Chaotic Systems
}

\author{
Chih-Hsueh Lin ${ }^{1}$, Chia-Wei Ho ${ }^{1,2}$, Guo-Hsin Hu ${ }^{1,2}$, Baswanth Sreeramaneni ${ }^{3}$ and Jun-Juh Yan $^{3, *(D)}$ \\ 1 Department of Electronic Engineering, National Kaohsiung University of Science and Technology, \\ Kaohsiung 80778, Taiwan; cslin@nkust.edu.tw (C.-H.L.); david80117@mail.mirdc.org.tw (C.-W.H.); \\ guohsin@mail.mirdc.org.tw (G.-H.H.) \\ 2 Department of Industrial Upgrading Service, Metal Industries Research \& Development Centre, \\ Kaohsiung 81160, Taiwan \\ 3 Department of Electronic Engineering, National Chin-Yi University of Technology, Taichung 41107, Taiwan; \\ baswanth101@gmail.com \\ * Correspondence: jjyan@ncut.edu.tw
}

Citation: Lin, C.-H.; Ho, C.-W.; Hu, G.-H.; Sreeramaneni, B.; Yan, J.-J. Secure Data Transmission Based on Adaptive Chattering-Free Sliding Mode Synchronization of Unified Chaotic Systems. Mathematics 2021, 9 , 2658. https://doi.org/10.3390/ math9212658

Academic Editor: Jan Awrejcewicz

Received: 6 September 2021

Accepted: 19 October 2021

Published: 21 October 2021

Publisher's Note: MDPI stays neutral with regard to jurisdictional claims in published maps and institutional affiliations.

Copyright: (c) 2021 by the authors. Licensee MDPI, Basel, Switzerland. This article is an open access article distributed under the terms and conditions of the Creative Commons Attribution (CC BY) license (https:/ / creativecommons.org/licenses/by/ $4.0 /)$.

\begin{abstract}
This paper is concerned with a novel secure data transmission design based on adaptive synchronization of master and slave unified chaotic systems. First, by introducing an augmented error state, an adaptive continuous sliding mode control (SMC) is derived to guarantee the synchronization of unified chaotic systems. Then, the secret message embedded in the master chaotic system can be transmitted from transmitter to receiver. Different from previous works using discontinuous SMC, the undesired chattering phenomenon can be fully eliminated, and it becomes possible to precisely recover the embedded secret message at the receiver. Last, an example is given to illustrate the success of secure data transmission with the continuous SMC developed in this paper.
\end{abstract}

Keywords: secure data transmission; chaos synchronization; adaptive sliding mode control; chattering; unified chaotic system

\section{Introduction}

Nowadays, chaos responses have been found in the dynamics of various engineering fields since the well-known Lorenz chaotic system described the dynamics of the atmosphere was proposed [1]. The dynamic behavior looks random, but it is quite different from the stochastic processes. Chaotic systems are with deterministic nonlinear dynamics to display the chaotic behavior. And there are many characteristics for chaotic systems, such as positive largest Lyapunov exponents, boundedness of strange attractors, sensitivity to initial value (so-called butterfly effect), broad spectrums of Fourier transform, random-like responses, fractal properties of states, and so forth [2,3]. Due to these characteristics of chaotic systems and their significant advantages in various engineering fields, the research on chaos suppression and synchronization control has attracted more and more attention. Chaos control is mainly to suppress chaotic behavior in the dynamic systems, while chaos synchronization introduces extra control input to force identical or different master-slave chaotic systems to have the same chaotic behavior. So far, researchers have used various control methods to solve the problems of chaos suppression or synchronization, such as H-infinity stabilization [4,5], adaptive control [6-9], robust SMC [10-14], backstepping control [15,16], optimal control [17,18], hybrid control [19], and so on. In the above research, the SMC has received special attention mainly due to attractive advantages, such as good performance of transient response, simple structure, easy implementation, and good robustness to external disturbances and parameter uncertainties. However, the chattering problem which can result in high-frequency oscillations in the controlled system needs to be well solved. An approach frequently introduced to solve the chattering phenomenon is to modify the designed discontinuous SMC with the boundary layer method [20-23]. 
However, it can only ensure the sliding motion outside a specified boundary layer, which will decrease the control performance and it is not suitable for the application to secure communication with high precision requirements. Zhang [24] proposed an integral sliding mode control (ISMC) method for a class of uncertain chaotic systems to eliminate the undesired chattering. However, it is necessary to use multi-dimensional inputs to complete the synchronization, which increases the complexity of the system design. Additionally, in the recent works [25-27], the authors utilized adaptive backstepping sliding mode control to effectively eliminate the chattering and successfully completed the actual engineering system control. However, their methods need further research before they can be extended to directly solve the problems of chaotic synchronization and secure communication considered in this paper.

In recent years, due to the importance of information security, many researchers have utilized the random property of chaotic systems to complete the design of secure communication [28-30]. The authors [28] utilized the advantages of fractional complex chaotic synchronization to design a secure communication. However, multi-dimensional inputs were needed to complete the synchronization. The authors $[29,30]$ proposed the method of chaotic masking, and the plaintext and chaotic signals are mixed into cipher text by addition. However, this masking encryption method is insecure, because the characteristics of the message are easily separated in the mixed encrypted signal.

Motivated by the aforementioned, this study aims to design an adaptive chatteringfree SMC to not only ensure the synchronization for master-slave unified chaotic dynamical systems but also achieve secure data transmission. First, we embed the secret information in the master chaotic system and ensure that its chaos dynamics are not destroyed. Then use the adaptive chattering-free SMC design to synchronize the master and slave chaotic systems, and precisely recover the secret information on the receiver. In the traditional $\mathrm{SMC}$, to ensure the hitting condition, the discontinuous sign function is often used to design the controller, but this function requires an infinite switching frequency in realization, so it will produce an undesirable chattering phenomenon. To eliminate chattering, as mentioned in [20-23], researchers introduced continuous saturation functions or boundary layers to replace the switching behavior of sign functions, but the accuracy performance of the control is lost, and certain errors of synchronization will be generated. In general control cases, this minor error due to chattering might be acceptable. However, this is a serious problem that must be solved in secure communication or encryption systems requiring high accuracy. Therefore, for secure communication, the continuous saturation function is not suitable for eliminating chattering. To remove the influence of chattering and accurately complete the secure data transmission, we introduced an augmented error state to derive an adaptive continuous SMC to guarantee the synchronization for master and slave unified chaotic systems without chattering. We introduce an augmented error state to construct a new switching function that contains only partial error states and ensure the convergence of the augmented error dynamics in the sliding manifold. Furthermore, the proposed control method only uses a single control input to complete synchronization control, which reduces the circuit complexity for SMC realization.

The rest of this study is organized as follows. Section 2 describes the dynamics of unified chaotic systems and formulates the chaos synchronization and secure data transmission problems. In Section 3, based on the SMC, an augmented error state is introduced, and a switching function is firstly proposed. Then the stability of the augmented error dynamics in the sliding manifold is discussed. In Section 4, an adaptive continuous SMC without involving any information of the secret message is established to ensure the sliding manifold with chattering. In Section 5, an illustrative example and comparisons are provided. Conclusions are provided in Section 6.

Notations: In this paper, $x^{T}$ is the transpose of the matrix $x ;|x|$ denotes the absolute value of $x ; \operatorname{sign}(\sigma)$ represents the sign function of $\sigma$ and $\operatorname{sign}(\sigma)=1$ when $\sigma>0 ; \operatorname{sign}(\sigma)=$ 0 when $\sigma=0 ; \operatorname{sign}(\sigma)=-1$ when $\sigma<0$. 


\section{System Description and Problem Formulation}

In this paper, we aim to design a secure data transmission with chaos synchronization using SMC. The transmitted secure data will be firstly embedded in the master chaotic system at the transmitter and can be safely and precisely recovered at the receiver. The secure information will not be exposed in public channels, so the information security can be ensured. We combine the characteristics of SMC and chaotic system synchronization to complete this design. In the following, we will consider the synchronization between master and slave unified chaotic systems with an adaptive chattering-free SMC. Although we use the unified chaotic system for discussion in this article, the method obtained can easily be extended to other types of chaotic systems.

\subsection{Unified Chaotic Systems}

A nonlinear unified chaotic system [31] is described by

$$
\begin{aligned}
& \dot{x}_{1}(t)=(25 \alpha+10)\left(x_{2}(t)-x_{1}(t)\right) \\
& \dot{x}_{2}(t)=(28-35 \alpha) x_{1}(t)+(29 \alpha-1) x_{2}(t)-x_{1}(t) x_{3}(t) \\
& \dot{x}_{3}(t)=x_{1}(t) x_{2}(t)-\frac{8+\alpha}{3} x_{3}(t)
\end{aligned}
$$

where $x_{1}, x_{2}, x_{3}$ denote the states and $\alpha$ is the given parameter with $0 \leq \alpha \leq 1$. System (1) can bridge the Lorentz attractor with Chen attractor and Lü attractor by the parameter $\alpha$. The unified system with $\alpha \in[0,0.8)$ belongs to the generalized Lorenz system and becomes the original Chen system when $\alpha \in(0.8,1]$. While $\alpha=0.8$, system (1) is the Lü system. The dynamics of the system (1) has been extensively researched in [28] and displays chaotic behavior in the whole interval $\alpha \in[0,1]$. Figure 1 demonstrates the bounded chaotic behaviors for $\alpha=0.3$ with initial values of $\left[\begin{array}{lll}x_{10} & x_{20} & x_{30}\end{array}\right]^{T}=\left[\begin{array}{lll}3 & 1 & 1\end{array}\right]^{T}$. Consequently, we will conclude a definite and simple procedure to design an adaptive chattering-free SMC to guarantee synchronization and complete the secure communication design.

(a)

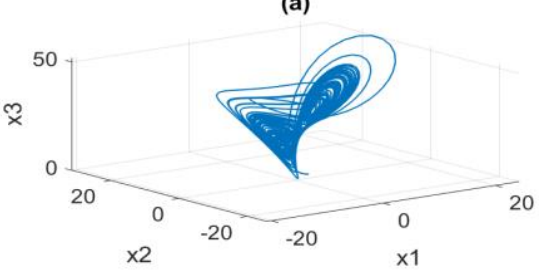

(c)

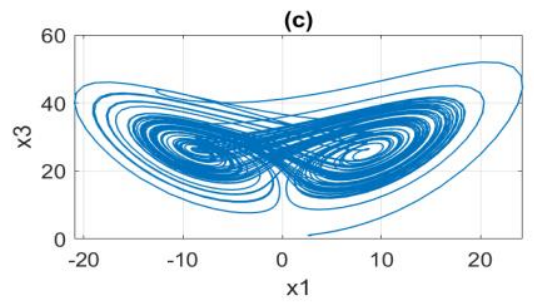

(b)

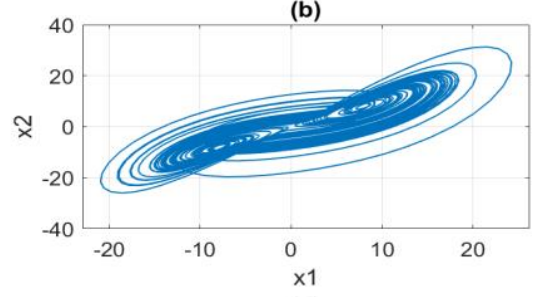

(d)

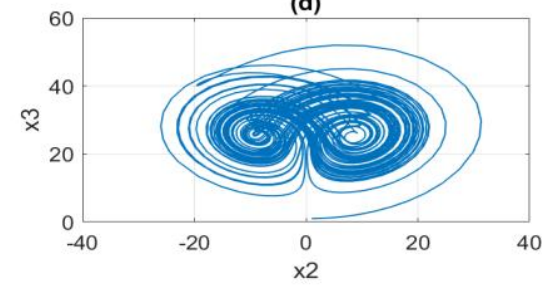

Figure 1. Bounded strange attractors of a unified chaotic system with $\alpha=0.3$ (a) $x_{1}-x_{2}-x_{3}$. (b) $x_{1}-x_{2}$ (c) $x_{1}-x_{3}$ (d) $x_{2}-x_{3}$.

\subsection{Communication Structure and Synchronization Problem Formulation}

Consider the structure of master and slave unified chaotic systems and the states of master and slave systems are denoted with $x$ and $y$, respectively.

$$
\begin{aligned}
& \dot{x}_{1}=(25 \alpha+10)\left(x_{2}-x_{1}\right) \\
& \dot{x}_{2}=(28-35 \alpha) x_{1}+(29 \alpha-1) x_{2}-x_{1} x_{3}+m(t) \\
& \dot{x}_{3}=x_{1} x_{2}-((8+\alpha) / 3) x_{3}
\end{aligned}
$$


and

$$
\begin{aligned}
& \dot{y}_{1}=(25 \alpha+10)\left(y_{2}-y_{1}\right) \\
& \dot{y}_{2}=(28-35 \alpha) y_{1}+(29 \alpha-1) y_{2}-y_{1} y_{3}+u(t) \\
& \dot{y}_{3}=y_{1} y_{2}-((8+\alpha) / 3) y_{3}
\end{aligned}
$$

where $x_{i}$ and $y_{i}, i=1,2,3$, respectively, denote the states of the master and slave systems. $m(t)$ is the embedded secret message and assumed that $m(t)$ and $\dot{m}(t)$ are bounded by $|m(t)| \leq \theta$, and $|\dot{m}(t)| \leq \rho$, where $\theta$ and $\rho$ are unknown positive constants. The $u(t) \in R$ in the slave system (3) is the introduced control input using adaptive chattering-free SMC to synchronize systems (2) and (3) and then achieve secure data transmission. By defining synchronization errors as follows:

$$
e_{i}(t)=y_{i}(t)-x_{i}(t), i=1,2,3
$$

then the error dynamics is obtained.

$$
\begin{aligned}
& \dot{e}_{1}(t)=(25 \alpha+10)\left(e_{2}(t)-e_{1}(t)\right) \\
& \dot{e}_{2}(t)=(28-35 \alpha) e_{1}(t)+(29 \alpha-1) e_{2}(t)-y_{1}(t) y_{3}(t)+x_{1}(t) x_{3}(t)-m(t)+u(t) \\
& \dot{e}_{3}(t)=-((8+\alpha) / 3) e_{3}(t)+y_{1}(t) y_{2}(t)-x_{1}(t) x_{2}(t)
\end{aligned}
$$

In this paper, with the slave system (3), a continuous adaptive SMC will be established without involving the unknown message $m(t)$ to synchronize the chaos responses of the slave system (3) with those of the master system (2) without chattering. Then the secret message $m(t)$ embedded in the master system (2) can be precisely recovered at the receiver (slave system), i.e.,

$$
\lim _{t \rightarrow \infty}\left\|e_{i}(t)\right\| \rightarrow 0, i=1,2,3 \text { and } \lim _{t \rightarrow \infty}(u(t)-m(t)) \rightarrow 0
$$

\section{Switching Function Design of SMC for Synchronization}

To guarantee the synchronization between systems (2) and (3) with the adaptive chattering-free SMC, an appropriate switching function must be firstly selected to result in $\lim _{t \rightarrow \infty}\left\|e_{i}(t)\right\| \rightarrow 0, i=1,2,3$ and $\lim _{t \rightarrow \infty}(u(t)-m(t)) \rightarrow 0$ when the augmented error dynamics enters into the sliding manifold. Then, an adaptive chattering-free SMC control law must be proposed to ensure the sliding manifold. To derive the continuous chattering-free $\mathrm{SMC}$, an augmented error state is newly introduced below.

$$
e_{a}(t)=(28-35 \alpha) e_{1}(t)+(29 \alpha-1) e_{2}(t)-y_{1}(t) y_{3}(t)+x_{1}(t) x_{3}(t)-m(t)+u(t)
$$

From Equation (7) and Equation (5), we have

$$
\begin{aligned}
\dot{e}_{a}(t) & =(28-35 \alpha) \dot{e}_{1}(t)+(29 \alpha-1) \dot{e}_{2}(t)-\left(\dot{y}_{1}(t) y_{3}(t)+y_{1}(t) \dot{y}_{3}(t)\right) \\
& +\left(\dot{x}_{1}(t) x_{3}(t)+x_{1}(t) \dot{x}_{3}(t)\right)-\dot{m}(t)+\dot{u}(t) \\
& =g(t)-\dot{m}(t)+\dot{u}(t)
\end{aligned}
$$

where

$$
\begin{aligned}
g(t)= & (28-35 \alpha)(25 \alpha+10)\left(e_{2}(t)-e_{1}(t)\right)+(29 \alpha-1) e_{a}(t) \\
& -(25 \alpha+10)\left(y_{2}(t) y_{3}(t)-x_{2}(t) x_{3}(t)\right)-y_{1}^{2}(t) y_{2}(t)+x_{1}^{2}(t) x_{2}(t) \\
& +\frac{38+76 \alpha}{3}\left(y_{1}(t) y_{3}(t)-x_{1}(t) x_{3}(t)\right)
\end{aligned}
$$

After introducing the augmented error state, the augmented error dynamics is rearranged as

$$
\begin{aligned}
& \dot{e}_{1}(t)=(25 \alpha+10)\left(e_{2}(t)-e_{1}(t)\right) \\
& \dot{e}_{2}(t)=e_{a}(t) \\
& \dot{e}_{a}(t)=g(t)-\dot{m}(t)+\dot{u}(t) \\
& \dot{e}_{3}(t)=-((8+\alpha) / 3) e_{3}(t)+y_{1}(t) y_{2}(t)-x_{1}(t) x_{2}(t)
\end{aligned}
$$


To complete the two major steps mentioned above for chattering-free SMC synchronization, a switching function is selected as

$$
\sigma(t)=e_{a}(t)+\int_{0}^{t}\left(k_{1} e_{1}(\tau)+k_{2} e_{2}(\tau)+k_{3} e_{a}(\tau)\right) d \tau
$$

where $\sigma(t) \in R$ and $k_{i}, i=1,2,3$ are designed parameters described later. When the error system (10) operates in the sliding manifold with $\sigma(t)=\dot{\sigma}(t)=0$ [10], from (11), one has

$$
\dot{e}_{a}(t)=-k_{1} e_{1}(t)-k_{2} e_{2}(t)-k_{3} e_{a}(t)
$$

Therefore, by Equation (10) and Equation (12), the following error dynamics in the sliding manifold is derived as

$$
\begin{aligned}
\overbrace{\left[\begin{array}{c}
\dot{e}_{1}(t) \\
\dot{e}_{2}(t) \\
\dot{e}_{a}(t)
\end{array}\right]}^{\dot{E}(t)} & =\left[\begin{array}{ccc}
-25 \alpha-10 & 25 \alpha+10 & 0 \\
0 & 0 & 1 \\
-k_{1} & -k_{2} & -k_{3}
\end{array}\right] \overbrace{\left[\begin{array}{c}
e_{1}(t) \\
e_{2}(t) \\
e_{a}(t)
\end{array}\right]}^{E(t)} \\
& =(\overbrace{\left[\begin{array}{ccc}
-25 \alpha-10 & 25 \alpha+10 & 0 \\
0 & 0 & 1 \\
0 & 0 & 0
\end{array}\right]}^{A}-\left[\begin{array}{l}
0 \\
0 \\
1
\end{array}\right] \overbrace{\left[\begin{array}{lll}
k_{1} & k_{2} & k_{3}
\end{array}\right]}^{K}]\left[\begin{array}{l}
e_{1}(t) \\
e_{2}(t) \\
e_{a}(t)
\end{array}\right]
\end{aligned}
$$

The controllability matrix $C=\left[\begin{array}{ccc}B & A B & A^{2} B\end{array}\right]=\left[\begin{array}{ccc}0 & 0 & 25 \alpha+10 \\ 0 & 1 & 0 \\ 1 & 0 & 0\end{array}\right]$ is with full row rank for all $0 \leq \alpha \leq 1$ and the $(A, B)$ in Equation (13) is a controllable matrix pair. Therefore, there always exists an appropriate matrix $K$ such that the eigenvalues $\lambda_{i}$ of the matrix $(A-B K)$ can be arbitrarily assigned to satisfy $\operatorname{Re}\left(\lambda_{i}\right)<0, i=1,2,3$ and $E(t)=\left[\begin{array}{lll}e_{1}(t) & e_{2}(t) & e_{a}(t)\end{array}\right]^{T}$ can converge to zero in finite time $t_{1}$. Furthermore, by Equation (10), when time $t \geq t_{1}, E(t)=0$ which implies $y_{1}(t) y_{2}(t)-x_{1}(t) x_{2}(t)=0$ for $t \geq t_{1}$. Consequently, the dynamics of $e_{3}(t)$ in Equation (13) degenerates to

$$
\dot{e}_{3}(t)=-((8+\alpha) / 3) e_{3}(t), \text { for } t \geq t_{1}
$$

Since $\alpha \in[0,1]$ and $(8+\alpha) / 3>0, e_{3}(t)$ also converges to zero. From the discussion above, the augmented error dynamics (10) in the sliding manifold is stable and ensure the synchronization.

Remark 1. From Equation (7), when $e_{a}(t)$ converges to zero, one has

$$
e_{a}(t)=(28-35 \alpha) e_{1}(t)+(29 \alpha-1) e_{2}(t)-y_{1}(t) y_{3}(t)+x_{1}(t) x_{3}(t)-m(t)+u(t)=0
$$

Furthermore, since all $e_{1}(t), e_{2}(t)$ and $e_{3}(t)$ converge to zero, the secret message $m(t)$ can be precisely recovered with $u(t)=m(t)$.

\section{Adaptive Continuous SMC Design without Chattering}

Having designed the switching function as Equation (11) and ensured the stability of the augmented error dynamics (10) in the sliding manifold, it is still necessary to give an adaptive continuous SMC to force the error dynamics (10) to hit and never escape the 
sliding manifold $\sigma(t)=0$. To guarantee the system trajectory can be controlled to the sliding manifold, the chattering-free adaptive SMC is given as

$$
\dot{u}(t)=-\left(g(t)+k_{1} e_{1}(t)+k_{2} e_{2}(t)+k_{3} e_{a}(t)+\mu \hat{\rho}(t) \operatorname{sign}(\sigma(t))\right) ; \mu>1
$$

or

$$
u(t)=u_{0}-\int_{0}^{t}\left(g(\tau)+k_{1} e_{1}(\tau)+k_{2} e_{2}(\tau)+k_{3} e_{a}(\tau)+\mu \hat{\rho}(\tau) \operatorname{sign}(\sigma(\tau))\right) d \tau ; \mu>1
$$

where $u_{0}$ is the initial condition of $u(t)$ and the adaptive law is given as follows:

$$
\dot{\hat{\rho}}(t)=|\sigma(t)|, \quad \hat{\rho}(0)=\hat{\rho}_{0}
$$

where $\hat{\rho}_{0}$ is the bounded initial condition of Equation (18).

Theorem 1. Consider the augmented error dynamics (10), if the control $u(t)$ in Equation (16) or Equation (17) with adaptive laws (18) is introduced, then the trajectory of systems can converge to the sliding manifold $\sigma(t)=0$.

To prove Theorem 1 , the following lemma is provided:

Lemma 1. (Barbalat lemma) [32]: If $w: R \rightarrow R$ is a uniformly continuous function for $t \geq 0$ and $\lim _{t \rightarrow \infty} \int_{0}^{t} w(\tau) d \tau$ exists and is bounded, then $\lim _{t \rightarrow \infty} w(t)=0$.

Proof of Theorem. Choose the continuous Lyapunov function $V(t)=\frac{1}{2}\left(\sigma^{2}(t)+\psi^{2}(t)\right) \geq$ 0 with $\psi(t)=\hat{\rho}(t)-\rho$ and $\dot{\psi}(t)=\dot{\hat{\rho}}(t)$, then

$$
\begin{aligned}
\dot{V}(t) & =\sigma(t) \dot{\sigma}(t)+\psi(t) \dot{\psi}(t) \\
& =\sigma(t)\left(\dot{e}_{a}(t)+k_{1} e_{1}(t)+k_{2} e_{2}(t)+k_{3} e_{a}(t)\right)+\psi(t) \dot{\hat{\rho}}(t) \\
& =\sigma(t)\left(g(t)-\dot{m}(t)+\dot{u}(t)+k_{1} e_{1}(t)+k_{2} e_{2}(t)+k_{3} e_{a}(t)\right)+\psi(t) \dot{\hat{\rho}}(t) \\
& =-\sigma(t) \dot{m}(t)-\sigma(t) \mu \hat{\rho}(t) \operatorname{sign}(\sigma(t))+\psi(t) \hat{\hat{\rho}}(t) \\
& \leq|\sigma(t)|(\rho-\hat{\rho}(t))+\hat{\rho}(t)|\sigma(t)|-\mu \hat{\rho}(t)|\sigma(t)|+\psi(t) \dot{\hat{\rho}}(t) \\
& \quad-\psi(t)
\end{aligned}
$$

where $|\dot{m}(t)| \leq \rho$. From Equation (19) and the adaptive law (18), we can conclude $\dot{V}(t) \leq(1-\mu) \hat{\rho}(t)|\sigma(t)|=-w(t) \leq 0$, where $w(t)=(\mu-1) \hat{\rho}(t)|\sigma(t)|$. Integrating $\dot{V}(t) \leq-w(t)$, it yields

$$
\begin{aligned}
& \int_{0}^{t} \dot{V}(\tau) d \tau \leq-\int_{0}^{t} w(\tau) d \tau \\
& \Rightarrow V(0) \geq V(t)+\int_{0}^{t} w(\tau) d \tau \geq \int_{0}^{t} w(\tau) d \tau
\end{aligned}
$$

Since $V(0)=\frac{1}{2}\left(\sigma^{2}(0)+\psi^{2}(0)\right)$ is always positive and bounded and $w(t) \geq 0$ for all time, $\lim _{t \rightarrow \infty} \int_{0}^{t} w(\tau) d \tau$ exists and is bounded. Therefore, by Barbalat's lemma [32], it ensures that

$$
\lim _{t \rightarrow \infty} w(t)=\lim _{t \rightarrow \infty}(\mu-1) \hat{\rho}(t)|\sigma(t)|=0
$$

Furthermore, since $\mu>1$ and $\hat{\rho}(t)>0$, we have $\sigma(t)=0$ as $t \rightarrow \infty$. The proof is completed.

Remark 2. The proposed adaptive SMC controller in Equations (16) and (17) demonstrates a continuous control input and chattering will not appear. 
Remark 3. Based on the above discussion, we conclude the design procedure for the secure data transmission based on adaptive SMC synchronization of unified chaotic systems as follows.

Step 1: Embed the secret data $m(t)$ into the master unified system (2).

Step 2: Introduce an augmented state as Equation (7) and construct the augmented error dynamics as Equation (10).

Step 3: Choose an appropriate matrix $K$ to make the eigenvalues $\lambda_{i}$ of $(A-B K)$ satisfy $\operatorname{Re}\left(\lambda_{i}\right)<$ $0, i=1,2,3$ to result in the stable sliding manifold.

Step 4: Establish the switching function $\sigma(t)$ in Equation (11).

Step 5: Construct the continuous SMC from Equation (16) or Equation (17) with the adaptive law (18).

Step 6: Achieve the robust synchronization and precisely recover the embedded secret message at the receiver.

\section{Numerical Simulations}

In this simulation, $\alpha=0$ is assigned such that system (1) becomes the original Lorenz system. The initial conditions $\left[\begin{array}{lll}x_{10} & x_{20} & x_{30}\end{array}\right]^{T}=\left[\begin{array}{lll}0.3 & -1 & -0.2\end{array}\right]^{T}$ and $\left[\begin{array}{lll}y_{10} & y_{20} & y_{30}\end{array}\right]^{T}=\left[\begin{array}{lll}-0.5 & 1 & 0.5\end{array}\right]^{T}$ are given. According to Remark 3 , the design is summarized as following steps:

Step 1: The secret data $m(t)=0.5 \sin (8 t)$ is embedded to the master system (2) with $\alpha=0$. Step 2: With $\alpha=0$ and the augmented state in Equation (15), the augmented error dynamics is obtained as

$$
\begin{aligned}
\dot{e}_{1}(t)= & 10\left(e_{2}(t)-e_{1}(t)\right) \\
\dot{e}_{2}(t)= & e_{a}(t) \\
\dot{e}_{a}(t)= & g(t)-\dot{m}(t)+\dot{u}(t) \\
\dot{e}_{3}(t)= & -(8 / 3) e_{3}(t)+y_{1}(t) y_{2}(t)-x_{1}(t) x_{2}(t) \\
g(t)= & (280)\left(e_{2}(t)-e_{1}(t)\right)-e_{a}(t) \\
& -10\left(y_{2}(t) y_{3}(t)-x_{2}(t) x_{3}(t)\right)-y_{1}^{2}(t) y_{2}(t)+x_{1}^{2}(t) x_{2}(t) \\
& +\frac{38}{3}\left(y_{1}(t) y_{3}(t)-x_{1}(t) x_{3}(t)\right)
\end{aligned}
$$

Step 3: Choose the matrix $K=\left[\begin{array}{lll}-0.8 & 44 & 13\end{array}\right]$ such that the eigenvalues of $(A-B K)$ are $\lambda_{1}=-9, \lambda_{2}=-8, \lambda_{3}=-6$ to result in a stable sliding manifold.

Step 4: Consequently, the switching function $\sigma(t)$ is constructed as:

$$
\sigma(t)=e_{a}(t)+\int_{0}^{t}\left(-0.8 e_{1}(\tau)+44 e_{2}(\tau)+13 e_{a}(\tau)\right) d \tau
$$

Step 5: From Equation (16) or Equation (17) with the adaptive law (18), the chattering-free adaptive SMC scheme with $\mu=1.2, u_{0}=1$ is obtained as

$$
\dot{u}(t)=-\left(g(t)-0.8 e_{1}(t)+44 e_{2}(t)+13 e_{a}(t)+1.2 \hat{\rho}(t) \operatorname{sign}(\sigma(t))\right)
$$

Or

$$
u(t)=1-\int_{0}^{t}\left(g(\tau)-0.8 e_{1}(\tau)+44 e_{2}(\tau)+13 e_{a}(\tau)+1.2 \hat{\rho}(\tau) \operatorname{sign}(\sigma(\tau))\right) d \tau
$$

the adaptive law is designed as $\dot{\hat{\rho}}(t)=|\sigma(t)|, \hat{\rho}(0)=0.1$.

The ode45 in the control toolbox of MATLAB is introduced. The short format of data type and step size 0.001 is selected for simulation. The simulation results with the continuous SMC (24) or (25) are given in Figures 2-4. Figure 2 shows the bounded strange attractor of the master Lorenz system with the embedded secret message $m(t)=0.5 \sin (8 t)$. The synchronization errors are given in Figure 3. Figure 4 shows the switching function $\sigma(t)$, the continuous control input $u(t)$, adaptation gain $\hat{\rho}(t)$ and the difference between $u(t)$ and $m(t)$. By observing the simulation results, the trajectories of error dynamics can 
converge to $\sigma(t)=0$ as expected and the synchronization is achieved. Additionally, the undesired chattering is fully eliminated due to the adaptive continuous SMC and the secret message is precisely recovered. To demonstrate the effectiveness of the proposed chattering-free controller, the continuous equivalent SMC proposed in Hou et al. [20] and Lin [21] is performed with the parameters $\eta=2, \beta=1, \psi=0.5$. To further compare with the traditional control, the parameter $\sigma$ in the equivalent controller proposed in $[20,21]$ is set to 0 and 0.02, respectively, and the simulation results are shown in Figures 5 and 6. In Figure 5, the parameter $\sigma$ is set to 0 and the equivalent controller becomes the traditional discontinuous controller. Observing the simulation results in Figure 5, it can be found that the chattering is obvious. Although there is a good performance in synchronization, the difference between the controller and message cannot converge due to the chattering, i.e., the transmitted secret message cannot be accurately recovered. In order to solve the problem of chattering, we choose the parameter $\sigma$ as 0.02. The simulation results in Figure 6 reveal that the chattering has been effectively eliminated, but the system cannot enter the sliding mode completely, so the performance of the control is affected and the transmitted message cannot be accurately recovered.

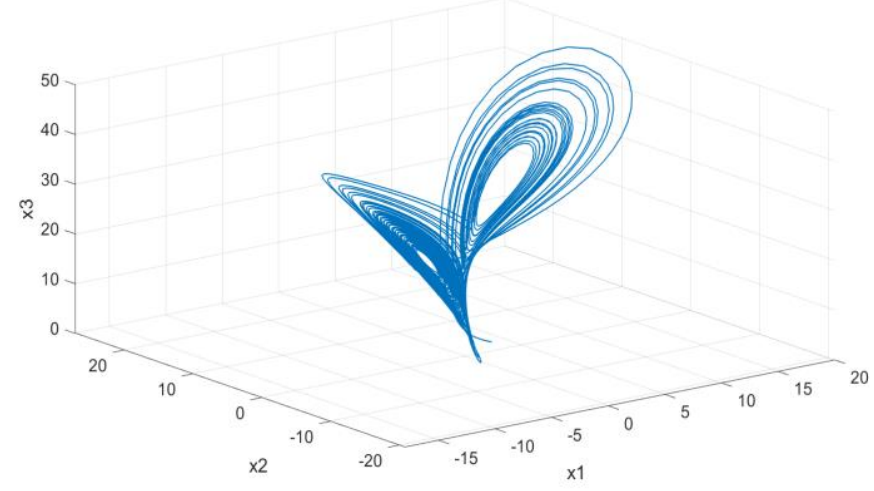

Figure 2. The bounded strange attractor of Lorenz system with the embedded message $m(t)=$ $0.5 \sin (8 t)$.
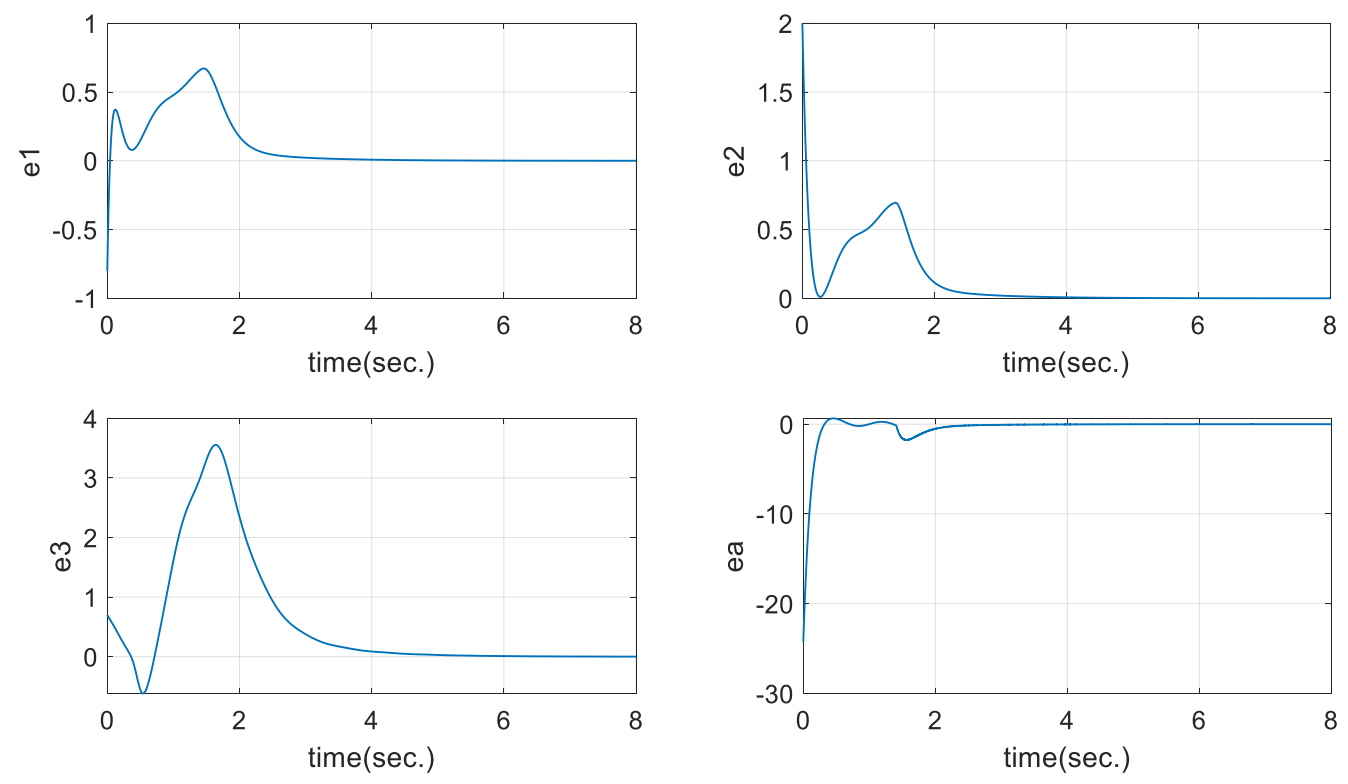

Figure 3. Error state responses. 

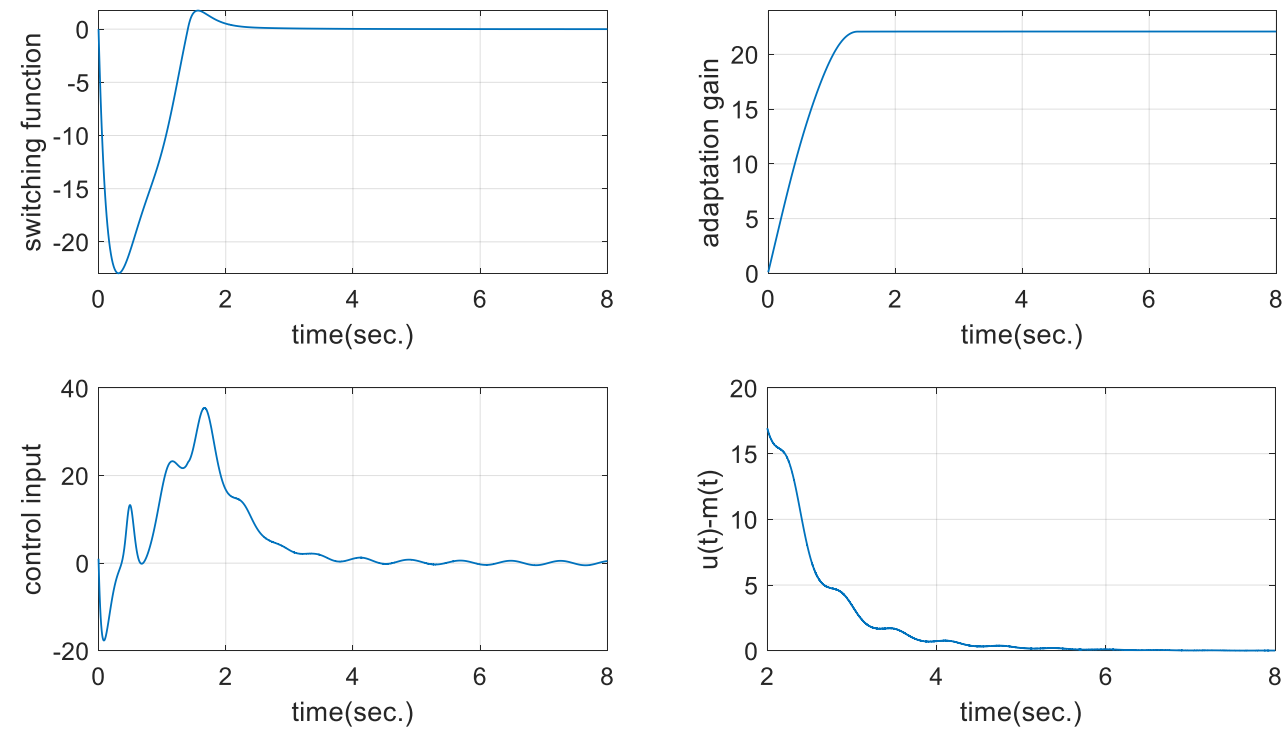

Figure 4. Controlled responses of switching function $\sigma(t)$, adaptation gain $\hat{\rho}(t)$, continuous control input $u(t)$ and the difference between $u(t)$ and $m(t)$.
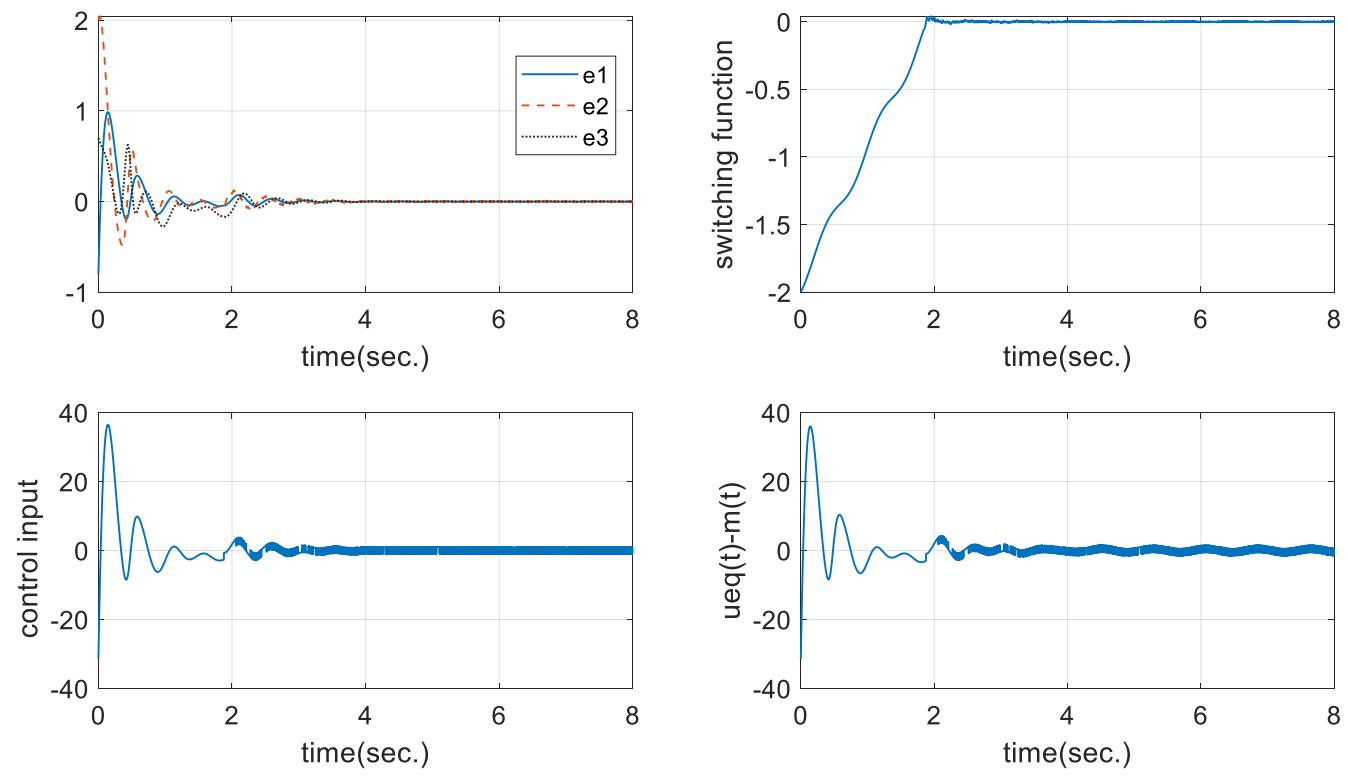

Figure 5. Controlled system responses with discontinuous SMC. 

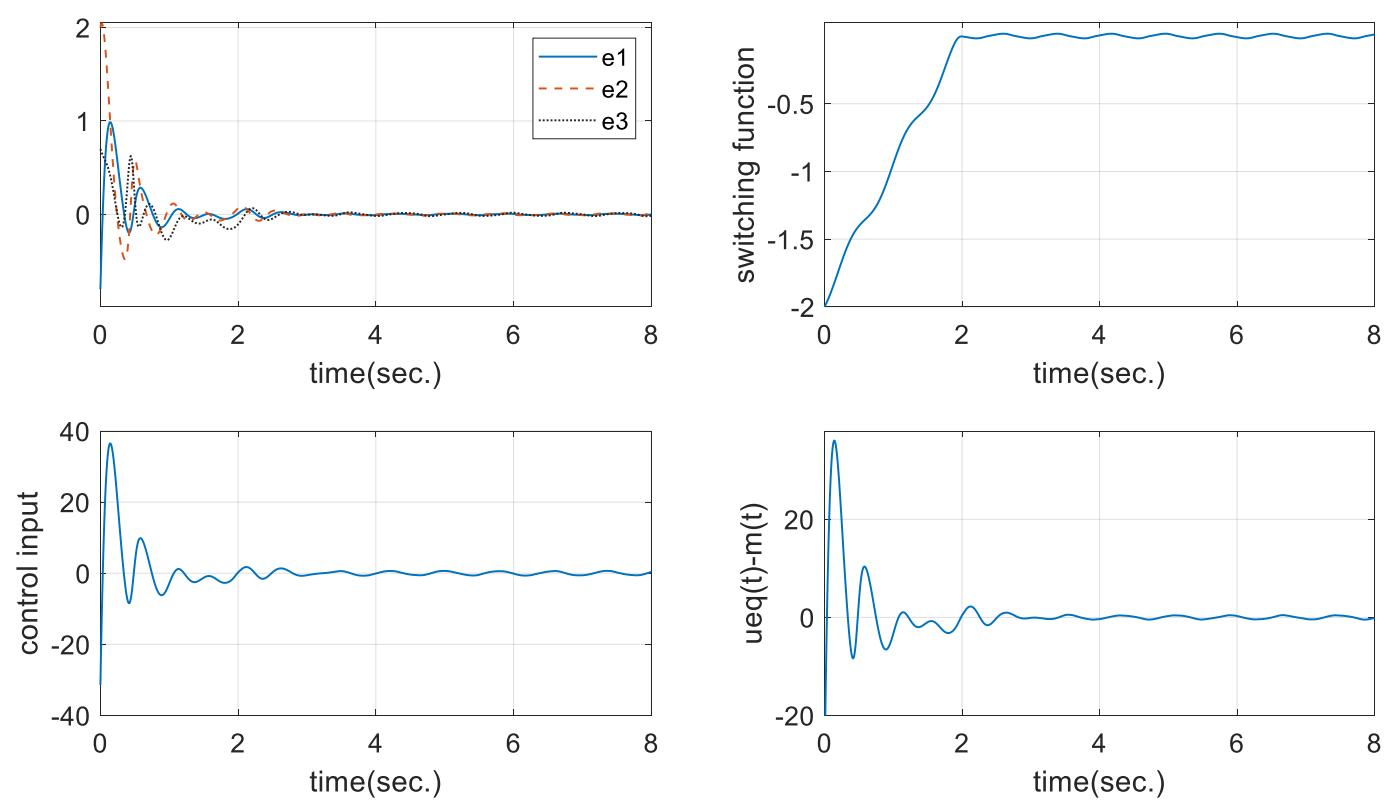

Figure 6. Controlled system responses with continuous equivalen SMC.

\section{Conclusions}

A secure data transmission approach based on adaptive synchronization of master and slave unified chaotic systems have been proposed. A new adaptive chattering-free SMC has been developed to synchronize the master and slave unified chaotic systems. According to the mathematical analysis and simulation results, we can observe that not only the synchronization can be ensured, but also the embedded secret message can be securely transmitted from transmitter to receiver. The numerical simulation results demonstrate the validity of the results developed in this paper. However, because this study only uses a single input for the synchronization control design, if one needs significantly speed up the synchronization, multi-dimensional inputs are necessary to achieve the fast synchronization, but it will increase the complexity of the controlled systems. Therefore, in the near future, extending the proposed chattering-free design to obtain finite-time synchronization is the proposed main work.

Author Contributions: All authors contributed to the paper. G.-H.H. wrote the manuscript with the supervision from C.-H.L. and J.-J.Y. G.-H.H., C.-W.H. and B.S. are responsible for the simulation of the robust chattering-free sliding mode control. All authors have read and agreed to the published version of the manuscript.

Funding: This work was financially supported by the Ministry of Science and Technology, Taiwan, under MOST- 110-2221-E-167 -030 and MOST-110-2218-E-006 -014 -MBK.

Institutional Review Board Statement: Not applicable.

Informed Consent Statement: Not applicable.

Data Availability Statement: Not applicable.

Conflicts of Interest: The authors declare no conflict of interest.

\section{References}

1. Lorenz, E.N. Deterministic nonperiodic flows. J. Atmos. Sci. 1963, 20, 130-141. [CrossRef]

2. Vanecek, A.; Celikovsky, S. Control Systems: From Linear Analysis to Synthesis of Chaos; Prentice-Hall International: London, UK, 1996.

3. Yau, H.T.; Chen, C.K.; Chen, C.L. Sliding mode control of chaotic systems with uncertainties. Int. J. Bifur. Chaos. 2000, 10, 1139-1147. [CrossRef] 
4. Lee, S.M.; Ji, D.H.; Park, J.H.; Won, S.C. Ho synchronization of chaotic systems via dynamic feedback approach. Phys. Lett. A 2008, 372, 4905-4912. [CrossRef]

5. Qin, Z.; Wang, J.L.; Huang, Y.L.; Ren, S.Y. Synchronization and H synchronization of multi-weighted complex delayed dynamical networks with fxed and switching topologies. J. Frankl. Inst. 2017, 354, 7119-7138. [CrossRef]

6. Gao, X.J.; Hu, H.P. Adaptive-impulsive synchronization and parameters estimation of chaotic systems with unknown parameters by using discontinuous drive signals. Appl. Math. Model. 2015, 39, 3980-3989. [CrossRef]

7. Tam, L.M.; Chen, H.K.; Li, S.Y. Adaptive synchronization of complicated chaotic systems with uncertainties via fuzzy modelingbased control strategy. Inf. Sci. 2018, 427, 18-31. [CrossRef]

8. Chen, X.Y.; Park, J.H.; Cao, J.D.; Qiu, J. Adaptive synchronization of multiple uncertain coupled chaotic systems via sliding mode control. Neurocomputing 2018, 273, 9-21. [CrossRef]

9. Boulkroune, A.; Bouzeriba, A.; Hamel, S.; Bouden, T. A projective synchronization scheme based on fuzzy adaptive control for unknown multivariable chaotic systems. Nonlinear Dyn. 2014, 78, 433-447. [CrossRef]

10. Yan, J.J.; Chen, C.Y.; Tsai, J.S.-H. Hybrid chaos control of continuous unified chaotic systems using discrete rippling sliding mode control, Nonlinear Analysis. Hybrid Syst. 2016, 22, 276-283. [CrossRef]

11. Muthana, T.A.; Mohamed, Z. Sliding Mode Control of Chaos in a Single Machine Connected to an Infinite Bus Power System. Math. Probl. Eng. 2018, 2018, 2703684.

12. Wang, C.C.; Yau, H.T. Nonlinear dynamic analysis and sliding mode control for a gyroscope system. Nonlinear Dynam. 2011, 66, 53-65. [CrossRef]

13. Vaidyanathan, S. Anti-synchronization of duffing double-well chaotic oscillators via integral sliding mode control. Int. J. ChemTech Res. 2016, 9, 297-304.

14. Singh, S. Single input sliding mode control for hyperchaotic Lu system with parameter uncertainty. Int. J. Dyn. Control 2016, 4, 504-514. [CrossRef]

15. Yu, Y.; Zhang, S. Controlling uncertain Lü system using backstepping design. Chaos Soltions Fractals 2003, 15, 897-902.

16. Njah, A.N. Tracking control and synchronization of the new hyperchaotic Liu system via backstepping techniques. Nonlinear Dynam. 2010, 61, 1-9. [CrossRef]

17. Yassen, M.T. The optimal control of Chen chaotic dynamical system. Appl. Math. Comput. 2002, 131, 171-180. [CrossRef]

18. Mohammad, A.; Arash, K.; Behzad, G. Control of chaos in permanent magnet synchronous motor by using optimal Lyapunov exponents placement. Phys. Lett. A 2010, 374, 4226-4230.

19. Fang, J.S.; Tsai, J.S.H.; Yan, J.J.; Chiang, L.H.; Guo, S.M. Hybrid H-infinity synchronization for uncertain continuous chaotic systems based on digital redesign approach. Meas. Control 2021. [CrossRef]

20. Hou, Y.Y.; Liau, B.Y.; Chen, H.C. Synchronization of Unified Chaotic Systems Using Sliding Mode Controller. Math. Probl. Eng. 2012, 2012, 632712. [CrossRef]

21. Lin, C.H.; Hu, G.H.; Yan, J.J. Chaos suppression in uncertain generalized lorenz-stenflo systems via a single rippling controller with input nonlinearity. Mathematics 2020, 8, 327. [CrossRef]

22. Giap, V.N.; Huang, S.C. Effectiveness of fuzzy sliding mode control boundary layer based on uncertainty and disturbance compensator on suspension active magnetic bearing system. Meas. Control 2020, 53, 934-942. [CrossRef]

23. Gandikota, G.; Das, D.K. Disturbance observer-based adaptive boundary layer sliding mode controller for a type of nonlinear multiple input multiple-output system. Int. J. Robust Nonlinear Control 2019, 29, 5886-5912. [CrossRef]

24. Zhang, H. An Integral Sliding Mode Control of Uncertain Chaotic Systems via Disturbance Observer. Complexity 2021, 2021, 6628116. [CrossRef]

25. Karami, M.; Kazemi, A.; Vatankhah, R.; Khosravifard, A. Adaptive fractional-order backstepping sliding mode controller design for an electrostatically actuated size-dependent microplate. J. Vib. Control 2021, 27, 1353-1369. [CrossRef]

26. Tuwa, R.N.; Woafo, P. Suppression of the noise-induced effects in an electrostatic micro-plate using an adaptive back-stepping sliding mode control. ISA Trans. 2018, 72, 100-109. [CrossRef]

27. Kingni, S.T.; Cheukem, A.; Tuwa, P.R.N.; Chamgoué, A.C.; Pham, V.T.; Jafari, S. Synchronous reluctance motor with load vibration perturbation: Analysis, electronic implementation and adaptive backstepping sliding mode control. Iran. J. Sci. Technol. Trans. Electr. Eng. 2021, 45, 645-654. [CrossRef]

28. Liu, J.; Wang, Z.; Shu, M.; Zhang, F.; Leng, S.; Sun, X. Secure Communication of Fractional Complex Chaotic Systems Based on Fractional Difference Function Synchronization. Complexity 2019, 2019, 7242791. [CrossRef]

29. Cuomo, K.M.; Oppenheim, A.V. Circuit implementation of synchronized chaos with applications to communications. Phys. Rev. Lett. 1993, 71, 65-68. [CrossRef]

30. Chen, J.; Li, C.; Yang, X. Chaos synchronization of the distributed-order Lorenz system via active control and applications in chaotic masking. Int. J. Bifurc. Chaos 2018, 28, 1850121-882. [CrossRef]

31. Lu, J.; Chen, G.; Cheng, D.; Celikovsk, S. Bridge the gap between the Lorenz system and the Chen system. Int. J. Bifurc. Chaos 2002, 12, 2917-2926. [CrossRef]

32. Popov, V.M. Hyperstability of Control System; Springer: Berlin/Heidelberg, Germany, 1973. 